\title{
The discovery and development of analgesics: new mechanisms, new modalities
}

\author{
Gillian Burgess and Dic Williams
}

Pain Research Unit, Pfizer Global Research and Development, Sandwich Laboratories, Sandwich, United Kingdom.

\begin{abstract}
Despite intensive research into pain mechanisms and significant investment in research and development, the majority of analgesics available to prescribers and patients are based on mechanistic classes of compounds that have been known for many years. With considerable ingenuity and innovation, researchers continue to make the best of the mechanistic approaches available, with novel formulations, routes of administration, and combination products. Here we review some of the mechanisms and modalities of analgesics that have recently entered into clinical development, which, coupled with advances in the understanding of the pathophysiology of chronic pain, will hopefully bring the promise of new therapeutics that have the potential to provide improved pain relief for those many patients whose needs remain poorly met.
\end{abstract}

\section{Introduction}

Drug discovery and development continue to be a challenge, with increasingly high investments in R\&D and increased numbers of submissions failing to translate into the delivery of novel chemical entities onto the market. The wide availability of generic and overthe-counter analgesics based on non-steroidal antiinflammatories (NSAIDs), acetaminophen, and "weak" opiates (and their combinations) provides many individuals with an accessible source of relief for mild to moderate pain. However, many patients with chronic conditions such as osteoarthritis remain poorly treated $(1,2)$.

Opiates continue to provide an important choice for the treatment of moderate to severe pain but are associated with a number of unwanted side effects such as constipation, nausea, vomiting, itching, and somnolence (3), and negative effects on patients' wellbeing, including sleep quality and ability to concentrate, which can be of such significance that they result in treatment discontinuation (4). Indeed, a recent Internet survey indicated that patients taking opiates for pain (and prescribing physicians) are willing to "trade-off" pain relief for a better toleration profile, with $50 \%$ of patients and physicians reporting that improved side effects represent the biggest unmet need (5).

Neuropathic pain has a complex pathophysiology and is difficult to treat (6). It has been estimated that about one-third of patients are likely to achieve 50\% pain relief with monotherapy (7); for example, in patients treated with the first-line therapy pregabalin, the patient global impression of change rating of much improved or very much improved was about $35 \%$ in postherpetic neuralgia, $50 \%$ in painful diabetic neuropathy, and $40 \%$ in fibromyalgia (8). A recent review identified a $66 \%$ increase in published randomized, placebo-controlled trials in a range of neuropathic pain populations over the last five years and concluded that only a limited improvement in the relief of pain had been achieved and that a large proportion of patients remained poorly treated (9).

The challenge for drug discovery and development can therefore be very simply stated: we need to bring forward analgesics that will provide more effective pain relief, are safe, and have a reduced sideeffect burden for a broader patient population.

Conflict of interest: Gillian Burgess and Dic Williams are employed by Pfizer. Citation for this article: J Clin Invest. 2010;120(11):3753-3759. doi:10.1172/JCI43195.

\section{Current strategies in advancing analgesics}

The approaches taken to select and advance analgesics through development and onto the market may be described in five broad categories. First, there are approaches designed to enhance the utility or increase the safety of existing medicines through the design of novel formulations or dosing forms. Examples include novel extended-release or abuse-resistant formulations of existing medicines such as oxycodone, morphine, and tramadol; and alternative delivery methods, such as patches for local administration of agents such as fentanyl, diclofenac, and lidocaine, as well as the oral fentanyl lozenge. Second, researchers have developed compounds directed against known mechanisms, especially of opiates and NSAIDs. Third, combination products of existing compounds have been developed, designed to improve efficacy or reduce unwanted side effects. Examples include combination products of oxycodone with ibuprofen, and naproxen in combination with the proton pump inhibitor esomeprazole (Vimovo). Fourth, there are a number of examples where the analgesic utility of medicines originally developed for other therapeutic indications has been recognized and led to products specifically directed toward the treatment of pain or where novel analgesics have been designed from known mechanistic classes of compounds. Examples of these include celecoxib, rofecoxib, pregabalin, duloxetine, ropivacaine, and tapentadol. Last - and largely in the minority - are therapeutics derived from the identification of novel mechanisms. These include the $\mathrm{N}$-type calcium channel blocker Prialt (ziconotide; Endo) and Sativex (GW Pharma), which has a cannabinoid-based mechanism. The numerical distribution of these approaches is summarized in Figure 1.

While recognizing the benefits of maximizing the utility of existing medicines, and based on the clear evidence that current medicines have failed to deliver significantly improved benefits (9), we conclude that more analgesics based on novel molecular mechanisms are necessary to address the unmet needs of patients in pain. The discovery and development of such medicines rely on an increased understanding of the pathophysiological mechanisms that drive pain and significant investment in $R \& D$ to translate this knowledge into novel, safe, and efficacious analgesics. Here, we describe a number of examples of emerging approaches and their mechanistic context. 


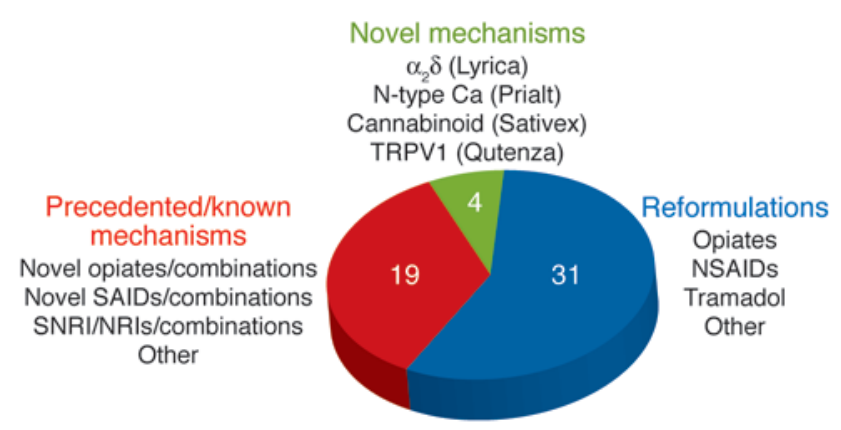

Figure 1

Analgesic launches from 1990 to 2010 . The majority of new drug launches were reformulations of existing pharmaceuticals. NRI, norepinephrine reuptake inhibitor; SAIDs, steroidal antiinflammatory drugs; SNRI, serotonin-norepinephrine reuptake inhibitor.

\section{Nerve growth factor}

It is now known that nerve growth factor (NGF), originally identified for its role in embryonic development of the nervous system $(10,11)$, has the capacity to alter the function of nociceptors in the adult long after it has ceased to be a requirement for neurotrophic maintenance (12-14). Exogenous administration of NGF induces pain in animals (15) and humans (16). NGF is released following tissue injury or inflammation from a range of cells including mast cells, macrophages, lymphocytes, fibroblasts, and keratinocytes and may become an important driver of pain symptoms in patients experiencing chronic pain, especially where inflammation is an important component. Proinflammatory actions of NGF have been described (17), and following burn or trauma it can result in release of inflammatory mediators (18), which in turn drive peripheral sensitization and pain. However, NGF may also act via direct effects on the sensory nerve endings themselves. Thus, in a study in healthy volunteers, intradermal injections of NGF induced local hypersensitivity with no associated neurogenic flare or inflammation (19). In this regard, it is interesting to note that it has been suggested that NGF released from keratinocytes following tissue damage may result in sensory hyperexcitability (20).

The directly affected population of nociceptors express the neurotrophin receptors tyrosine kinase receptor $\mathrm{A}(\operatorname{Trk} \mathrm{A})$ and/or $\mathrm{p} 75$, which mediate the actions of NGF (21) via both transcriptional and nontranscriptional mechanisms to increase excitability. Relatively rapid effects of high levels of NGF on nociceptors include the increased phosphorylation of key ion channels such as transient receptor potential vanilloid 1 (TRPV1), $\mathrm{Na}_{v} 1.7$, or $\mathrm{Na}_{v} 1.8$, thus increasing excitability and decreasing the threshold for firing (22). NGF bound to its receptor complex is internalized, and a proportion is transported retrogradely to the cell body of the sensory nerves within dorsal root ganglia, where, via regulators such as NF- $\mathrm{KB}$ or CREB, it upregulates expression of ion channels such as $\mathrm{Na}_{v} 1.8$ and TRPV1 $(22,23)$ and peptide transmitters (24-26). Upregulation of brain-derived neurotrophic factor (BDNF) might also occur in response to NGF, and it is interesting to speculate that this could contribute to central sensitization via actions on TrkB receptors on post-synaptic neurons in the dorsal horn of the spinal cord (27).

A number of clinical programs are being pursued with a view to bringing therapeutics to patients based on the sequestration of NGF or the inhibition of the activation mechanism via TrkA. The most advanced of these is a humanized monoclonal antibody, tanezumab (RN-624, PF-04383119) (28), derived from the murine anti-NGF monoclonal antibody muMab-911 (29). The humanized monoclonal antibody is being studied in a range of phase II and phase III clinical trials to evaluate its efficacy, safety, and tolerability in patients with a number of painful syndromes. These include osteoarthritis of the knee or hip, chronic low back pain, interstitial cystitis/painful bladder syndrome, and cancer bone pain, endometriosis, chronic prostatitis, and neuropathic pain (30). Phase II data are yet to be published, and the antibody is being further evaluated in phase III clinical studies of osteoarthritis patients as monotherapy in NSAID failures, in combination studies with NSAIDs, and studies to directly compare the therapeutic utility versus NSAIDs or opioids. Extensive evaluation is ongoing to determine the benefits and risks of this new therapeutic approach.

The current thinking on the pivotal role played by elevated NGF in driving peripheral sensitization such as that occurring in chronic inflammatory conditions is reflected in investment by a number of pharmaceutical companies in the development of monoclonal antibody approaches against NGF. These are summarized in Table 1 and discussed below.

The Johnson \& Johnson corporation is exploring the efficacy, safety, and tolerability of the human anti-NGF antibody JNJ42160443 (formerly AMJ-403) in a number of phase II studies as adjunctive therapy in subjects with moderate to severe knee or hip pain from osteoarthritis, subjects with inadequately controlled moderate to severe chronic low back pain, and patients with chronic bladder pain from interstitial cystitis and/or painful bladder syndrome, painful diabetic neuropathy, cancer pain, and postherpetic or post-traumatic neuralgia (31).

\section{Table 1}

Clinical programs in development utilizing NGF monoclonal antibodies

\begin{tabular}{|c|c|c|c|c|}
\hline Drug name & Manufacturer & Pain type & Trial phase & Ref. \\
\hline $\begin{array}{l}\text { Tanezumab } \\
\text { (RN-624, PF-04383119) }\end{array}$ & Pfizer & $\begin{array}{l}\text { Osteoarthritis of the knee or hip, chronic low back pain, } \\
\text { interstitial cystitis, endometriosis, chronic prostatitis } \\
\text { with pelvic pain syndrome }\end{array}$ & II/III & 30 \\
\hline JNJ 42160433 & Johnson \& Johnson & $\begin{array}{c}\text { Chronic low back pain, cancer, diabetic neuropathy, } \\
\text { postherpetic neuralgia, interstitial cystitis }\end{array}$ & II & 31 \\
\hline REGN475 & Regeneron/Sanofi Aventis & $\begin{array}{l}\text { Osteoarthritis of the knee, sciatica, chronic pancreatitis, } \\
\text { non-traumatic vertebral fracture, thermal injury }\end{array}$ & $\mathrm{I} / \mathrm{II}$ & 32 \\
\hline PG110 & Abbot Laboratories & Osteoarthritis of the knee & I & 34 \\
\hline
\end{tabular}




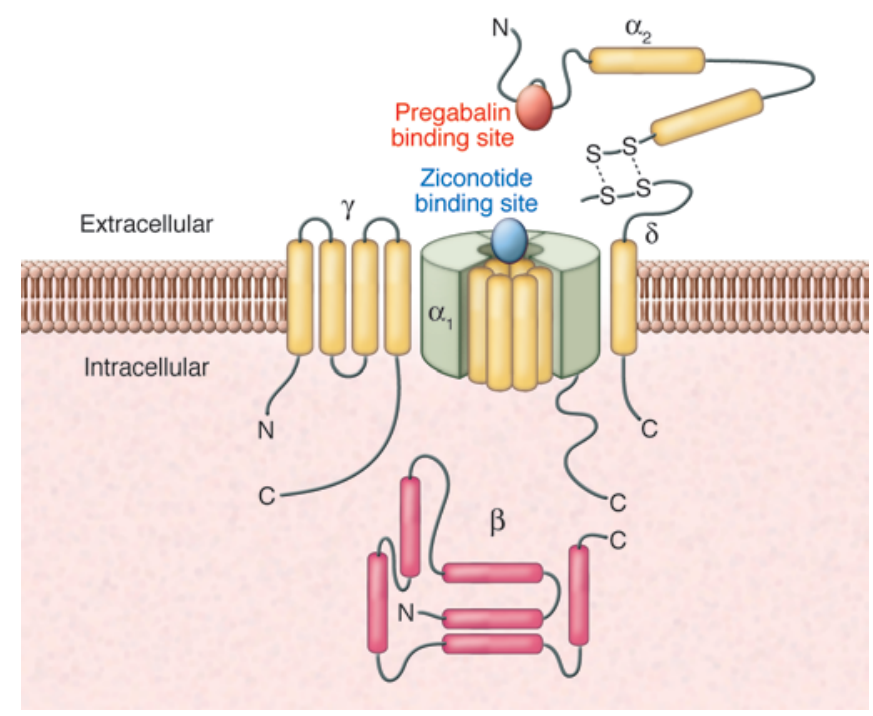

Figure 2

Schematic diagram showing the relative binding sites of ziconotide and pregabalin to voltage-gated calcium channel complex. Ziconotide binds to the pore-forming $\alpha_{1}$ subunit, whereas pregabalin binds to the accessory subunit $\alpha_{2} \delta$. Figure adapted with permission from Sigma-Aldrich.

Regeneron Pharmaceuticals, in collaboration with Sanofi Aventis, is conducting a phase I/II randomized, double blind, placebocontrolled, parallel-group, repeat-dose study of the safety and efficacy of REGN475 in patients with osteoarthritis of the knee. The antibody will be given in two intravenous doses. The company is also recruiting for a series of phase II studies to explore efficacy in patients with sciatica, moderate to severe abdominal pain due to chronic pancreatitis of at least 6 months duration, moderate to severe pain due to nontraumatic vertebral fracture, and pain due to thermal injury (32). Similarly, MedImmune has announced a study to assess the potential of MEDI-578 in patients with osteoarthritis of the knee (33). At the time of writing, Abbot Laboratories is recruiting into a phase I clinical trial to evaluate the safety and tolerability of a monoclonal antibody to NGF (PG110) in patients with osteoarthritis of the knee. The single-dose study will assess the pain score of subjects as part of the secondary assessments (34).

It is exciting that, depending on the outcome of a number of safety and efficacy studies, we might look forward to the possible emergence of a novel mechanistic approach and therapeutic modality with several antibody-based therapeutics targeting the NGF system over the next few years. In the future, these approaches may be followed by antibodies (35) and or small molecules that bind to the TrkA complex.

\section{Targeting ion channels}

Sodium channels. The pain-killing effects of extracts derived from the coca leaf have been recognized for millennia, and it has been known for decades that drugs refined from the active ingredient cocaine and developed as alternatives, such as procaine, lidocaine, and bupivacaine, can provide powerful analgesia and be used as local anesthetics (36). It is now known that these compounds act by blocking voltage-gated sodium channels, and the high efficacy is consistent with the critical role of these channels in the generation and propagation of action potentials (37). However, sodium channels subserve this critical role in all excitable cells, including cardiac cells, and local anesthetics are nonselective in their actions. Thus, the use of systemic analgesics of this class is limited, and the developmental focus has been to maximize their therapeutic potential with topical approaches. The development of patch technologies has expanded the utility of local anesthetics, and they are used successfully in some neuropathic pain conditions such as postherpetic neuralgia $(38,39)$ and the control of postoperative pain (40), although not in HIV-associated pain (41).

Anticonvulsants such as lamotrigine, phenytoin, and carbamazepine, acting in the central nervous system, have been used in the treatment of chronic pain for a number of years (42). It is known that they all bind to sodium channels preferentially in conditions of high neuronal excitability (43). There has been considerable interest and investment in recent years in approaches based on the selective targeting of specific sodium channel subtypes (44). Some nine different subtypes have been identified $\left(\mathrm{Na}_{v} 1.1-1.9\right)$, differentiated on the basis of their $\alpha$ subunits (45). A number of these channels have been shown to be present in human nociceptors, primarily $\mathrm{Na}_{\mathrm{v}} 1.7,-1.8$, and 1.9 , and their expression is precisely regulated (46). The attention has thus far been on the search for subtype-specific sodium channel blockers of $\mathrm{Na}_{\mathrm{v}} 1.8$ and $\mathrm{Na}_{\mathrm{v}}$ 1.7. The rationale is supported by an impressive and growing body of human genetic data, such as the linkage of Nav1.7 mutations to extreme positive and negative pain phenotypes $(47,48)$, and extensive mouse knockout data that have emerged in recent years (49). However, this effort has yet to result in any compound emerging from human clinical trials. It has been suggested that one reason for the lack of progress has been that the discovery strategies have included primary screens that tend to select compounds that bind at, or very close to, the pore-forming regions of the channel complex (50). This region is highly conserved between subtypes and constitutes the site at which local anesthetics bind (51). Emerging data from studies on the actions of a range of peptide toxins provide some encouragement that targeting non-pore regions may be possible, but it seems that it may be some time before a safe and truly selective subtype-specific sodium channel ligand emerges onto the market.

Calcium channels. Calcium channels are key to neuronal function, particularly in regulating neurotransmitter release from nerve terminals, including those that transmit pain at the level of the spinal cord. Ziconotide (52-54) is a synthetic peptide equivalent to the venom of the sea snail Conus magus. The compound, termed a conopeptide, blocks $\mathrm{N}$-type voltage-gated calcium channels and is likely effective as an analgesic through an action that prevents transmission across the first synapse in the pain pathway. Consistent with the mechanism of action, the compound is effective in patients with intractable pain when delivered either alone or in combination with other analgesics and in whom intrathecal therapy is acceptable (52, $55,56)$. Maximal therapeutic benefit requires careful titration in order to minimize the range of CNS side effects associated with its use. These include dizziness, confusion, ataxia, memory deficits, and hallucinations (56). The compound must be administered using specified infusion protocols and patients kept under close supervision (55). Leconotide is an alternative conopeptide, and animal data have been described suggesting that it can be delivered systemically with fewer CNS side effects than ziconotide (57). Given the wide distribution and central role of voltage-gated calcium channels in neurotransmission, approaches based on channel blockade will 
likely continue to face significant challenges in terms of therapeutic index, but where this can be managed, they may provide an important option for the treatment of severe intractable pain.

Ziconotide blocks calcium entry through the channel by binding to a specific region of the pore-forming $\alpha$-subunit (58). Pregabalin and its predecessor gabapentin, which are effective in the treatment of neuropathic pain (8), interact with voltage-gated calcium channels in a different way than ziconotide (Figure 2). These compounds bind to the calcium channel accessory subunit $\alpha_{2} \delta$ (59), and it has been shown that binding to this subunit is a requirement for the analgesic efficacy of pregabalin (60). However, a greater understanding of the role of the subunit in neuronal function, its involvement in pain pathophysiology, and how the binding of pregabalin to this protein delivers efficacy in neuropathic pain conditions may lead to novel ways to modulate this mechanism and to new therapeutic approaches.

It has been shown that $\alpha_{2} \delta$ protein increases in both dorsal horn and dorsal root ganglia following peripheral nerve injury in animal models of neuropathic pain (61), suggesting that peripheral nerve injury may increase the number of calcium channels in the sensory presynaptic terminal. Indeed, evidence has been presented that the $\alpha_{2} \delta$ protein may be involved in the trafficking of calcium channel complexes into the membrane $(62,63)$ and that pregabalin or gabapentin may act by blocking this increase (64). This raises the interesting possibility that future therapeutic approaches based on targeting the mechanisms regulating trafficking of ion channels could be developed.

More recently it has been suggested that increased synaptogenesis occurring in the spinal cord following peripheral nerve injury may involve the binding of thrombospondin to $\alpha_{2} \delta-1$ and that gabapentin and pregabalin (65) may reduce central hypersensitivity by blocking this interaction. However, it is not clear whether active synaptogenesis or increased synaptic turnover is occurring in pain pathways in patients with stable neuropathic pain at presentation, and in whom pregabalin is therapeutically effective. Further work is therefore necessary to fully explore the significance of the thrombospondin pathway in driving pain conditions and its potential as a novel therapeutic approach for chronic pain. A recent study involving biochemical and immunocytochemical approaches suggests that the $\alpha_{2} \delta$ subunit may be anchored on the outside of the plasma membrane (66). This provides both a challenge and an opportunity to further explore the potential interactions of the $\alpha_{2} \delta$ subunit with the pore-forming complex of calcium channels and the consequences of pregabalin binding and may lead to the design of improved pain therapeutics acting through this mechanism.

$G A B A$. It has been suggested that central sensitization may, at least in part, be the result of an effective decrease in central inhibitory mechanisms. GABA receptors are key ligand-gated chloride channels present on both pre- and postsynaptic neurons and important in the regulation of pain transmission. Benzodiazepines are GABA receptor agonists that are used as part of the treatment of, for example, chronic low back pain, but usefulness as an analgesic is limited by unwanted CNS side effects (67). However, the potential for GABA-A-mediated analgesia is indicated by the efficacy of spinally administered benzodiazepines (68). Studies using a series of knockout mice suggest that selective activation of a subset of GABA receptors may deliver analgesia with a reduced CNS adverse effect liability (69). Subtype-selective agonists have been advanced into development for treatment of anxiety but none have yet emerged as potential analgesics (70).
Transient receptor potential channels. There has been significant scientific interest and industry investment in recent years to understand the function of the broad family of nonspecific cation TRPV channels in transduction and transmission mechanisms. The first to be described was TRPV1, and the belief that it acts as an integrator of a variety of sensitization processes in the periphery has driven significant focus in attempts to identify molecules that inactivate the channel. It is now some years since the emergence of capsazepine (71) as the first TRPV1 antagonist, but despite significant investment, there are no definitive clinical studies demonstrating the effectiveness and safety of compounds that block or inactivate this channel (72). It is clear that one major stumbling block has been the effects of TRPV1 blockers on core body temperature. Thus, in one study, the Amgen compound AMG 517 administered following third molar extraction resulted in significant hyperthermia, with maximal body temperature exceeding $40^{\circ} \mathrm{C}(73,74)$.

Much remains to be understood about the role of the channel in this physiological phenomenon, but preclinical data suggest that therapeutic strategies based on exclusion of compounds from the CNS may not be sufficient, since some of the actions on thermoregulation reside in the periphery, and it is likely that some of the analgesic effects may be mediated by actions at spinal nociceptive synapses $(75,76)$. The identification of compounds that pharmacologically distinguish between the analgesic and hyperthermic effects of TRPV1 antagonism will be required to explore fully the therapeutic potential of this channel for the treatment of pain. Indeed, TRPV1 antagonists (e.g., AMG8562) have recently been identified that are reported to have no effect on body temperature (77).

An alternative approach to exploit the mechanism and avoid side effects associated with systemic exposure has been to develop topical preparations (78-80). In order to avoid the acute irritation, capsaicin $(8 \%)$ is administered following application of topical lidocaine and is reported to be well tolerated following repeated administration (81). It is interesting to note that the mechanism of action may involve both the desensitization of the peripheral nociceptor endings and a decrease in epidermal fiber density (78).

Other TRP channels, especially the non-desensitizing TRPV3, have received interest for their therapeutic potential (82), and Glenmark's TRPV3 agonist GRC 15300 is the first molecule to enter clinical trials (83).

Earlier preclinical interest in TRPA1 was driven by studies in mutant mice suggesting a role for the channel in mechanotransduction (84) and potentially noxious cold or cold allodynia $(85,86)$, and it seems that some of these actions may be mediated via central mechanisms. It has been suggested that this channel may be a key mediator in specific inflammatory pathways such as those involving phospholipase C (87). More recently, a rare human gain-of-function mutation has been identified that results in an increase in channel function at normal membrane potential and is associated with an episodic pain syndrome characterized by exaggerated hypersensitivity (88).

\section{Mechanisms based on activated microglia}

In recent years, there has been much interest in the potential role of non-neuronal cells in the pathophysiology of chronic pain. These include microglia, astrocytes, and oligodendrocytes, and considerable efforts have been made to understand the molecular basis of their interaction with neuronal pain processing, especially at the level of the spinal cord. A number of excellent reviews of the experimental evidence gathered from animal models of chronic pain, especially those involving nerve damage, have been published $(89,90)$. 
The thesis recognizes that the pathophysiology of neuropathic pain includes not only increased and chronic neurotransmitter activation of the neuronal pain pathway, but also activation of glial cells, altering their status in such a way that leads to the release of chemokines, cytokines, protease enzymes, and trophic factors, which in turn alter the excitability of the neuronal elements in the pathway (89). Intriguingly, evidence has been published indicating that opiates may also activate these non-neuronal elements and that this may reduce their therapeutic benefit via mediation of tolerance and respiratory depression (90).

It has been suggested that one of the consequences of glial activation is the downregulation of the $\mathrm{K}^{+} \mathrm{Cl}^{-}$transporter $\mathrm{KCC} 2$ mediated by the release of BDNF, which results in the erosion of the chloride gradient across membranes of postsynaptic cells $(91,92)$. Thus, inhibitory mechanisms that rely on the opening of chloride channels would be reversed or more likely reduced by glial activation (93). If this phenomenon occurs in patients, it would provide a rationale for a therapeutic approach based on restoration of KCC2 function, a fascinating if challenging endeavor (94).

Despite considerable research into the role of activated glia in the development of hypersensitive states in animal models, there remains little known of the temporal, anatomical, cytological, and pathophysiological role of these phenomena and their relation to the development and progression of pain symptoms in patients. Some insight may be gained by the application of imaging techniques such as the use of PET ligands at the peripheral benzodiazepine receptor to studies of the temporal pattern of "activation" in patients, but the true therapeutic opportunities offered by this phenomenon can only be realized by well-designed clinical trials coupled to validated biomarkers in patients. Two such examples of pioneering studies in this area involve the compounds ibudilast (95) and propentofylline (96). Propentofylline attenuates pain behaviors in rodent models of neuropathic pain $(97,98)$ and has been shown to modulate inhibitory tone following spinal injury (99) by reducing glial activation via alterations in glutamate promoter activation (96).

Ibudilast reduces the release of proinflammatory factors such as cytokines from activated microglia in culture (100) and reduces pain responses in experimental animals (101). Both ibudilast and propentofylline have been entered into clinical trials to evaluate their therapeutic potential $(102,103)$. However, to date, no positive data have emerged from these studies.

p38 MAP kinase is thought to be a key mediator of glial activation (see ref. 89). In neuropathic conditions, fractalkine, released from neurons, activates the receptor CX3CR1 on microglia, and this results in phosphorylation of p38 and microglial activation. At the time of writing, participants are being recruited for a phase II study to evaluate a $\mathrm{p} 38$ inhibitor in patients experiencing neuropathic pain following nerve trauma (104). The study is scheduled to be completed in December 2010.

\section{Cannabinoids}

Cannabis has long been thought to bring benefit to patients experiencing chronic pain, but a recent review of randomized controlled trials concluded that the evidence indicated that the efficacy and tolerability of cannabinoids remained questionable and that they should be used as adjunctive agents where appropriate (105).
Sativex is a cannabinoid approved in Canada as an adjunctive analgesic treatment in patients with opiate-resistant cancer pain and for the relief of neuropathic pain in multiple sclerosis and is reported to provide statistically significant pain relief and improved sleep when given in addition to existing medication to patients with neuropathic pain $(106,107)$ or rheumatoid arthritis $(108)$. Its use in pain management has been reviewed recently (109).

The objective in maximizing the medicinal value of cannabinoids for pain has been to optimize the analgesic benefits while minimizing the unwanted central side effects. Two cannabinoid receptors have been cloned (CB1 and CB2) and a number of proposed endocannabinoid ligands identified. These include anandamide (AEA) (110) and 2-arachidonylglycerol (2-AG) (111). The enzymes fatty acid amide hydrolase (FAAH) (112) and monoacylglycerol lipase (MAGL) (113) are the primary catabolic enzymes of AEA and 2-AG, respectively, and are key in the regulation of the functional availability of these endogenous ligands. One therapeutic rationale is based on the possibility that endocannabinoids may provide an important component of the endogenous control of pain transmission and eventually perception, and that increasing their availability by specifically blocking the catabolizing enzymes may result in analgesia with minimal CNS side effects. Indeed, it has been shown in experimental animals that blockade of FAAH reduces pain responses without substantially affecting motility or cognition (114). Recently, an FAAH inhibitor with high selectivity that covalently inactivates the enzyme by carbamylation of the serine nucleophile has been identified (115). The compound completely inhibits FAAH activity and results in significantly elevated brain AEA levels. An FAAH inhibitor is currently in phase II development for osteoarthritis (116) and will also be evaluated in other conditions where cannabinoids have been shown to bring some pain relief. We must await the outcome of these trials to fully appreciate the analgesic efficacy available from maximizing the endogenous pain control delivered by this mechanism.

\section{Conclusion}

Despite significant investment in pain research, the challenges facing the development of novel therapeutics remain significant. The needs of patients for more effective pain relief are unlikely to be met unless new mechanisms are added to the analgesic armamentarium. Exciting new approaches are emerging, and the increasing application of genetic approaches and increased investment in pain research have helped identify what we hope will be high-confidence pain targets based on a sound understanding of mechanisms. However, drug discovery remains a very high-risk endeavor, and the time and costs of discovering and developing the compounds, as well as the methodologies to translate the research effort into medicines that better meet the needs of people in pain, remain a significant challenge.

\section{Acknowledgments}

We thank Kenneth Verburg for reading the manuscript.

Address correspondence to: Gillian Burgess, Pfizer Global Research and Development, Ramsgate Road, Sandwich, Kent, CT13 9NJ United Kingdom. Phone: 44.1304.646057; Fax: 44.1304.658444; E-mail: Gillian.Burgess@Pfizer.com.
1. Bjordal JM, Klovning A, Ljunggren AE, Slordal L. Short-term efficacy of pharmacotherapeutic interventions in osteoarthritic knee pain: a metaanalysis of randomised placebo-controlled trials.
Eur J Pain. 2007;11(2):125-138.

2. Moore RA, Derry S, Makinson GT, McQuay HJ. Tolerability and adverse events in clinical trials of celecoxib in osteoarthritis and rheumatoid arthritis: systematic review and meta-analysis of information from company clinical trial reports. Arthritis Res Ther. 2005;7(3):R644-R665.

3. Kalso E, Edwards JE, Moore RA, McQuay HJ. Opi- 
oids in chronic non-cancer pain: systematic review of efficacy and safety. Pain. 2004;112(3):372-380.

4. Chou R, et al. Clinical guidelines for the use of chronic opioid therapy in chronic noncancer pain. J Pain. 2009;10(2):113-130.

5. Gregorian RS Jr, Gasik A, Kwong WJ, Voeller S Kavanagh S. Importance of side effects in opioid treatment: a trade-off analysis with patients and physicians [published online ahead of print May 7, 2010]. J Pain. doi: 10.1016/j.jpain.2010.02.007.

6. Dworkin RH, et al. Pharmacologic management of neuropathic pain: evidence-based recommendations. Pain. 2007;132(3):237-251.

7. Gottrup H, Bach FW, Juhl G, Jensen TS. Differential effect of ketamine and lidocaine on spontaneous and mechanical evoked pain in patients with nerve injury pain. Anesthesiology. 2006;104(3):527-536.

8. Moore RA, Straube S, Wiffen PJ, Derry S, McQuay $\mathrm{HJ}$. Pregabalin for acute and chronic pain in adults. Cochrane Database Syst Rev. 2009;(3):CD007076.

9. Finnerup NB, Sindrup SH, Jensen TS. The evidence for pharmacological treatment of neuropathic pain. Pain. 2010;150(3):573-581.

10. Levi-Montalcini R. Effects of mouse tumor transplantation on the nervous system. Ann NY Acad Sci. 1952;55(2):330-344.

11. Levi-Montalcini R. The nerve growth factor 35 years later. Science. 1987;237(4819):1154-1162.

12. Pezet S, McMahon SB. Neurotrophins: mediators and modulators of pain. Annu Rev Neurosii 2006;29:507-538.

13. Watson JJ, Allen SJ, Dawbarn D. Targeting nerve growth factor in pain: what is the therapeutic potential? BioDrugs. 2008;22(6):349-359.

14. Nicol GD, Vasko MR. Unraveling the story of NGF-mediated sensitization of nociceptive sensory neurons: ON or OFF the Trks? Mol Interv. 2007; $7(1): 26-41$

15. Lewin GR, Rueff A, Mendell LM. Peripheral and central mechanisms of NGF-induced hyperalgesia. Eur J Neurosci. 1994;6(12):1903-1912.

16. Svensson P, Cairns BE, Wang K, Arendt-Nielsen L. Injection of nerve growth factor into human masseter muscle evokes long-lasting mechanical allodynia and hyperalgesia. Pain. 2003;104(1-2):241-247.

17. Aloe L, Levi-Montalcini R. Mast cells increase in tissues of neonatal rats injected with the nerve growth factor. Brain Res. 1977;133(2):358-366

18. Woolf CJ, Safieh-Garabedian B, Ma QP, Crilly P, Winter J. Nerve growth factor contributes to the generation of inflammatory sensory hypersensitivity. Neuroscience. 1994;62(2):327-331.

19. Rukwied R, Mayer A, Kluschina O, Obreja O, Schley M, Schmelz M. NGF induces non-inflammatory localized and lasting mechanical and thermal hypersensitivity in human skin. Pain. 2010; 148(3):407-413.

20. Radtke C, Vogt PM, Devor M, Kocsis JD. Keratinocytes acting on injured afferents induce extreme neuronal hyperexcitability and chronic pain. Pain. 2010;148(1):94-102.

21. Zampieri N, Chao MV. Mechanisms of neurotrophin receptor signalling. Biochem Soc Trans. 2006 34(pt 4):607-611.

22. Black JA, Liu S, Tanaka M, Cummins TR, Waxman SG. Changes in the expression of tetrodotoxinsensitive sodium channels within dorsal root ganglia neurons in inflammatory pain. Pain. 2004; 108(3):237-247.

23. Toledo-Aral JJ, Brehm P, Halegoua S, Mandel G. A single pulse of nerve growth factor triggers longterm neuronal excitability through sodium channel gene induction. Neuron. 1995;14(3):607-611.

24. McMahon SB, Bennett DL, Priestley JV, Shelton DL. The biological effects of endogenous nerve growth factor on adult sensory neurons revealed by a trkAIgG fusion molecule. Nat Med. 1995;1(8):774-780.

25. Donnerer J, Schuligoi R, Stein C. Increased con- tent and transport of substance $\mathrm{P}$ and calcitonin gene-related peptide in sensory nerves innervating inflamed tissue: evidence for a regulatory function of nerve growth factor in vivo. Neuroscience. 1992;49(3):693-698.

26. Woolf CJ. Phenotypic modification of primary sensory neurons: the role of nerve growth factor in the production of persistent pain. Philos Trans R Soc Lond B Biol Sci. 1996;351(1338):441-448.

27. Pezet S, Malcangio M, McMahon SB. BDNF: a neuromodulator in nociceptive pathways? Brain Res Brain Res Rev. 2002;40(1-3):240-249.

28. Cattaneo A. Tanezumab, a recombinant humanized $\mathrm{mAb}$ against nerve growth factor for the treatment of acute and chronic pain. Curr Opin Mol Ther. 2010;12(1):94-106

29. Hongo JS, et al. Antibody binding regions on human nerve growth factor identified by homolog- and alanine-scanning mutagenesis. Hybridoma. 2000;19(3):215-227.

30. 23 Studies into the safety and efficacy of tanezumab in pain patients. Clinicaltrials.gov Web Site. http:// www.clinicaltrial.gov/ct2/results?term=tanezumab. Accessed March 23, 2010

31. Studies evaluating the safety and efficacy of JNJ42160442 in pain patients. Clinicaltrials.gov NIH Web Site. http://www.clinicaltrial.gov/ct2/res ults?term=JNJ42160443. Accessed March 23, 2010

32. Studies evaluating the safety and efficacy of REGN475 in pain patients. Clinicaltrials.gov NIH Web Site. http://www.clinicaltrial.gov/ct2/ results?term=REGN475. Accessed March 23, 2010.

33. Study to Assess MEDI-578 in Patients With Osteoarthritis $(\mathrm{OA})$ of the Knee. Clinicaltrials.gov NIH Web Site. http://www.clinicaltrial.gov/ct2/show/ NCT01072591?term=medi-578rank=1. Accessed March 24, 2010.

34. Safety and Tolerability of PG110 in Patients With Knee Osteoarthritis Pain. Clinicaltrials.gov NIH Web Site. http://www.clinicaltrial.gov/ct2/ results?term=PG110. Accessed March 23, 2010.

35. BioXell and Lay Line Genomics announce license agreement for anti-TrkA antibody against pain [press release]. Rome, Italy. February 13, 2006.

36. Harris A, Goldberg LG. Spinal anaesthesia with nupercaine and procaine: a comparative study. Ann Surg. 1931;94(5):934-938.

37. Catterall WA. From ionic currents to molecular mechanisms: the structure and function of voltagegated sodium channels. Neuron. 2000;26(1):13-25.

38. Rowbotham MC, Davies PS, Verkempinck C, Galer BS. Lidocaine patch: double-blind controlled study of a new treatment method for post-herpetic neuralgia. Pain. 1996;65(1):39-44.

39. Galer BS, Jensen MP, Ma T, Davies PS, Rowbotham MC. The lidocaine patch $5 \%$ effectively treats all neuropathic pain qualities: results of a randomized, double-blind, vehicle-controlled, 3-week efficacy study with use of the neuropathic pain scale. Clin J Pain. 2002;18(5):297-301.

40. Saber AA, Elgamal MH, Rao AJ, Itawi EA, Martinez RL. Early experience with lidocaine patch for postoperative pain control after laparoscopic ventral hernia repair. Int J Surg. 2009;7(1):36-38

41. Estanislao L, Carter K, McArthur J, Olney R, Simpson D. A randomized controlled trial of 5\% lidocaine gel for HIV-associated distal symmetric polyneuropathy. J Acquir Immune Defic Syndr. 2004;37(5):1584-1586

42. Wiffen PJ, McQuay HJ, Moore RA. Carbamazepine for acute and chronic pain. Cochrane Database Syst Rev. 2005;(3):CD005451.

43. Lipkind GM, Fozzard HA. Molecular model of anticonvulsant drug binding to the voltagegated sodium channel inner pore. Mol Pharmacol. 2010;78(4):631-638

44. England S, de Groot MJ. Subtype-selective targeting of voltage-gated sodium channels. BrJ Pharma- col. 2009;158(6):1413-1425

45. Catterall WA, Goldin AL, Waxman SG. International Union of Pharmacology. XLVII. Nomenclature and structure-function relationships of voltage-gated sodium channels. Pharmacol Rev. 2005;57(4):397-409.

46. Rush AM, Cummins TR, Waxman SG. Multiple sodium channels and their roles in electrogenesis within dorsal root ganglion neurons. J Physiol. 2007;579(pt 1):1-14.

47. Drenth JP, Waxman SG. Mutations in sodium-channel gene SCN9A cause a spectrum of human genetic pain disorders. J Clin Invest. 2007;117(12):3603-3609.

48. Dib-Hajj SD, Cummins TR, Black JA, Waxman SG. Sodium channels in normal and pathological pain. Annu Rev Neurosci. 2010;33:325-347.

49. Lampert A, O’Reilly AO, Reeh P, Leffler A. Sodium channelopathies and pain. Pflugers Arch. 2010;460(2):249-263.

50. Bhattacharya A, Wickenden AD, Chaplan SR. Sodium channel blockers for the treatment of neuropathic pain. Neurotherapeutics. 2009;6(4):663-678.

51. England S. Voltage-gated sodium channels: the search for subtype-selective analgesics. Expert Opin Investig Drugs. 2008;17(12):1849-1864.

52 . Wallace MS. Ziconotide: a new nonopioid intrathecal analgesic for the treatment of chronic pain. Expert Rev Neurother. 2006;6(10):1423-1428.

53. Smith HS, Deer TR. Safety and efficacy of intrathecal ziconotide in the management of severe chronic pain. Ther Clin Risk Manag. 2009;5(3):521-534.

54. Miljanich GP. Ziconotide: neuronal calcium channel blocker for treating severe chronic pain. Curr Med Chem. 2004;11(23):3029-3040.

55. Schmidtko A, Lotsch J, Freynhagen R, Geisslinger G. Ziconotide for treatment of severe chronic pain. Lancet. 2010;375(9725):1569-1577.

56. Rauck RL, et al. A randomized, double-blind, placebo-controlled study of intrathecal ziconotide in adults with severe chronic pain. J Pain Symptom Manage. 2006;31(5):393-406.

57. Kolosov A, Goodchild CS, Cooke I. CNSB004 (Leconotide) causes antihyperalgesia without side effects when given intravenously: a comparison with ziconotide in a rat model of diabetic neuropathic pain. Pain Med. 2010;11(2):262-273.

58. Feng ZP, Hamid J, Doering C, Bosey GM, Snutch TP, Zamponi GW. Residue Gly1326 of the N-type calcium channel alpha $1 \mathrm{~B}$ subunit controls reversibility of omega-conotoxin GVIA and MVIIA block. J Biol Chem. 2001;276(19):15728-15735.

59. Dooley DJ, Taylor CP, Donevan S, Feltner D. $\mathrm{Ca} 2+$ channel alpha2delta ligands: novel modulators of neurotransmission. Trends Pharmacol Sci. 2007;28(2):75-82.

60 . Field MJ, et al. Identification of the alpha2-delta-1 subunit of voltage-dependent calcium channels as a molecular target for pain mediating the analgesic actions of pregabalin. Proc Natl Acad Sci U S A. 2006;103(46):17537-17542.

61. Li CY, Song YH, Higuera ES, Luo ZD. Spinal dorsal horn calcium channel alpha2delta-1 subunit upregulation contributes to peripheral nerve injury-induced tactile allodynia. J Neurosci. 2004;24(39):8494-8499

62 . Hendrich J, et al. Pharmacological disruption of calcium channel trafficking by the alpha2delta ligand gabapentin. Proc Natl Acad Sci U S A. 2008;105(9):3628-3633.

63. Bauer CS, et al. The increased trafficking of the calcium channel subunit alpha2delta-1 to presynaptic terminals in neuropathic pain is inhibited by the alpha2delta ligand pregabalin. J Neurosci. 2009;29(13):4076-4088

64. Bauer CS, Rahman W, Tran-van-Minh A, Lujan R, Dickenson AH, Dolphin AC. The anti-allodynic alpha(2)delta ligand pregabalin inhibits the traf- 
ficking of the calcium channel alpha(2)delta-1 subunit to presynaptic terminals in vivo. Biochem Soc Trans. 2010;38(2):525-528.

65. Eroglu C, et al. Gabapentin receptor alpha2delta-1 is a neuronal thrombospondin receptor responsible for excitatory CNS synaptogenesis. Cell. 2009;139(2):380-392.

66. Davies A, et al. The alpha2delta subunits of voltage-gated calcium channels form GPI-anchored proteins, a posttranslational modification essential for function. Proc Natl Acad Sci U S A. 2010; 107(4):1654-1659.

67. Chou R. Pharmacological management of low back pain. Drugs. 2010;70(4):387-402

68. Jasmin L, Wu MV, Ohara PT. GABA puts a stop to pain. Curr Drug Targets CNS Neurol Disord. 2004;3(6):487-505

69. Munro G, Ahring PK, Mirza NR. Developing analgesics by enhancing spinal inhibition after injury: GABAA receptor subtypes as novel targets. Trends Pharmacol Sci. 2009;30(9):453-459.

70. Lewis RT, et al. A pyridazine series of alpha2/alpha3 subtype selective GABA A agonists for the treatment of anxiety. J Med Chem. 2006;49(8):2600-2610.

71. Bevan S, et al. Capsazepine: a competitive antagonist of the sensory neurone excitant capsaicin. $\mathrm{BrJ}$ Pharmacol. 1992;107(2):544-552.

72. Szallasi A, Cortright DN, Blum CA, Eid SR. The vanilloid receptor TRPV1:10 years from channel cloning to antagonist proof-of-concept. Nat Rev Drug Discov. 2007;6(5):357-372.

73. Gavva NR, et al. Pharmacological blockade of the vanilloid receptor TRPV1 elicits marked hyperthermia in humans. Pain. 2008;136(1-2):202-210.

74. Romanovsky AA, et al. The transient receptor potential vanilloid-1 channel in thermoregulation: a thermosensor it is not. Pharmacol Rev. 2009;61(3):228-261.

75. Cui M, et al. TRPV1 receptors in the CNS play a key role in broad-spectrum analgesia of TRPV1 antagonists. J Neurosci. 2006;26(37):9385-9393.

76. Tamayo N, et al. Design and synthesis of peripherally restricted transient receptor potential vanilloid 1 (TRPV1) antagonists. J Med Chem 2008;51(9):2744-2757.

77. Lehto SG, et al. Antihyperalgesic effects of (R,E)-N(2-hydroxy-2,3-dihydro-1H-inden-4-yl)-3-(2-(piperidin-1-yl)-4-(tri fluoromethyl)phenyl)-acrylamide (AMG8562), a novel transient receptor potentia vanilloid type 1 modulator that does not cause hyperthermia in rats. J Pharmacol Exp Ther. 2008; 326(1):218-229

78. Kennedy WR, et al. A randomized, controlled, open-label study of the long-term effects of NGX 4010, a high-concentration capsaicin patch, on epidermal nerve fiber density and sensory function in healthy volunteers. J Pain. 2010;11(6):579-587.

79. Backonja M, et al. NGX-4010, a high-concentration capsaicin patch, for the treatment of postherpetic neuralgia: a randomised, double-blind study. Lancet Neurol. 2008;7(12):1106-1112.

80. Simpson DM, Brown S, Tobias J. Controlled trial of high-concentration capsaicin patch for treatment of painful HIV neuropathy. Neurology. 2008;70(24):2305-2313.

81. Simpson DM, et al. Long-term safety of NGX-4010, a high-concentration capsaicin patch, in patients with peripheral neuropathic pain. J Pain Symptom
Manage. 2010;39(6):1053-1064.

82. Bevan S, Andersson DA. TRP channel antagonists for pain - opportunities beyond TRPV1. Curr Opin Investig Drugs. 2009;10(7):655-663.

83. Glenmark's TRPV3 agonist GRC 15300 is the first molecule globally to enter clinical trials. Glenmark Web Site. http://glenmarkpharma.com/UITemplate/HtmlContainer.aspx?res=P_GLN_GDY_ AOVR. Accessed March 24, 2010.

84. Petrus $M$, et al. A role of TRPA1 in mechanical hyperalgesia is revealed by pharmacological inhibition. Mol Pain. 2007;3:40.

85. Bautista DM, et al. TRPA1 mediates the inflammatory actions of environmental irritants and proalgesic agents. Cell. 2006;124(6):1269-1282.

86. Katsura H, et al. Antisense knock down of TRPA1, but not TRPM8, alleviates cold hyperalgesia after spinal nerve ligation in rats. Exp Neurol. 2006; 200(1):112-123.

87. Eid SR, Cortright DN. Transient receptor potential channels on sensory nerves. Handb Exp Pharmacol. 2009;(194):261-281.

88. Kremeyer B, et al. A gain-of-function mutation in TRPA1 causes familial episodic pain syndrome. Neuron. 2010;66(5):671-680.

89. McMahon SB, Malcangio M. Current challenges in glia-pain biology. Neuron. 2009;64(1):46-54.

90. Watkins LR, Hutchinson MR, Rice KC, Maier SF. The "toll" of opioid-induced glial activation: improving the clinical efficacy of opioids by targeting glia. Trends Pharmacol Sci. 2009;30(11):581-591.

91 . Coull JA, et al. Trans-synaptic shift in anion gradient in spinal lamina I neurons as a mechanism of neuropathic pain. Nature. 2003;424(6951):938-942.

92. Coull JA, et al. BDNF from microglia causes the shift in neuronal anion gradient underlying neuropathic pain. Nature. 2005;438(7070):1017-1021.

93. Prescott SA, Sejnowski TJ, De Koninck Y. Reduction of anion reversal potential subverts the inhibitory control of firing rate in spinal lamina I neurons: towards a biophysical basis for neuropathic pain. Mol Pain. 2006;2:32

94. Coull JA, Gagnon M. The manipulation of cationchloride co-transporters as a novel means to treat persistent pain, epilepsy and other neurological disorders. Curr Opin Investig Drugs. 2009;10(1):56-61.

95. Rolan P, Hutchinson M, Johnson K. Ibudilast: a review of its pharmacology, efficacy and safety in respiratory and neurological disease. Expert Opin Pharmacother. 2009;10(17):2897-2904.

96. Tawfik VL, et al. Propentofylline-induced astrocyte modulation leads to alterations in glial glutamate promoter activation following spinal nerve transection. Neuroscience. 2008;152(4):1086-1092.

97. Sweitzer SM, Schubert P, DeLeo JA. Propentofylline, a glial modulating agent, exhibits antiallodynic properties in a rat model of neuropathic pain. J Pharmacol Exp Ther. 2001;297(3):1210-1217.

98. Sweitzer SM, Pahl JL, DeLeo JA. Propentofylline attenuates vincristine-induced peripheral neuropathy in the rat. Neurosci Lett. 2006;400(3):258-261.

99. Gwak YS, Crown ED, Unabia GC, Hulsebosch CE. Propentofylline attenuates allodynia, glial activation and modulates GABAergic tone after spinal cord injury in the rat. Pain. 2008;138(2):410-422.

100.Mizuno T, et al. Neuroprotective role of phosphodiesterase inhibitor ibudilast on neuronal cell death induced by activated microglia. Neurophar macology. 2004;46(3):404-411.

101. Ledeboer A, et al. The glial modulatory drug AV411 attenuates mechanical allodynia in rat models of neuropathic pain. Neuron Glia Biol. 2006;2(4):279-291.

102. Sheridan C. Glial cells on the radar. Nat Biotechnol. 2009;27(2):114-116.

103. Safety, Tolerability, Pharmacokinetics and Preliminary Efficacy of AV411 in Neuropathic Pain. Clinicaltrials.gov NIH Web Site. http://www. clinicaltrial.gov/ct2/show/NCT00576277?term= AV411 rank=2. Accessed July 27, 2010.

104.A Randomised, Double Blind Study to Evaluate the Safety and Efficacy of the p38 Kinase Inhibitor, GW856553, in Subjects With Neuropathic Pain From Peripheral Nerve Injury. Clinicaltrials.gov NIH Web Site. http://www.clinicaltrial.gov/ct2/ show $/$ NCT00969059?term $=p+38$ rank=7. Accessed March 30, 2010.

105. Turcotte D, Le Dorze JA, Esfahani F, Frost E, Gomori A, Namaka M. Examining the roles of cannabinoids in pain and other therapeutic indications: a review. Expert Opin Pharmacother. 2010; 11(1):17-31.

106. Berman JS, Symonds C, Birch R. Efficacy of two cannabis based medicinal extracts for relief of central neuropathic pain from brachial plexus avulsion: results of a randomised controlled trial. Pain. 2004;112(3):299-306.

107. Nurmikko TJ, Serpell MG, Hoggart B, Toomey PJ, Morlion BJ, Haines D. Sativex successfully treats neuropathic pain characterised by allodynia: a randomised, double-blind, placebo-controlled clinical trial. Pain. 2007;133(1-3):210-220.

108. Blake DR, Robson P, Ho M, Jubb RW, McCabe CS. Preliminary assessment of the efficacy, tolerability and safety of a cannabis-based medicine (Sativex) in the treatment of pain caused by rheumatoid arthritis. Rheumatology (Oxford). 2006;45(1):50-52.

109. Perez J, Ribera MV. Managing neuropathic pain with Sativex: a review of its pros and cons. Expert Opin Pharmacother. 2008;9(7):1189-1195.

110.Devane WA, et al. Isolation and structure of a brain constituent that binds to the cannabinoid receptor. Science. 1992;258(5090):1946-1949.

111. Mechoulam R, et al. Identification of an endogenous 2-monoglyceride, present in canine gut, that binds to cannabinoid receptors. Biochem Pharmacol. 1995;50(1):83-90.

112. Cravatt BF, Giang DK, Mayfield SP, Boger DL, Lerner RA, Gilula NB. Molecular characterization of an enzyme that degrades neuromodulatory fatty-acid amides. Nature. 1996;384(6604):83-87.

113. Blankman JL, Simon GM, Cravatt BF. A comprehensive profile of brain enzymes that hydrolyze the endocannabinoid 2-arachidonoylglycerol. Chem Biol. 2007;14(12):1347-1356.

114. Kinsey SG, et al. Blockade of endocannabinoiddegrading enzymes attenuates neuropathic pain. J Pharmacol Exp Ther. 2009;330(3):902-910.

115.Ahn K, et al. Discovery and characterization of a highly selective FAAH inhibitor that reduces inflammatory pain. Chem Biol. 2009;16(4):411-420.

116. A Study to Investigate Whether PF-04457845 Is Effective in Treating Pain, Is Safe and Tolerable in Patients with Osteoarthritis of the Knee. Clinicaltrials.gov NIH Web Site. http://clinicaltrials.gov/ct2/ show/NCT00981357?term=Pf-04457845rank=1. Accessed March 24, 2010 